Teknokultura. Revista de Cultura Digital y Movimientos Sociales

ISSNe: $1549-2230$

http://dx.doi.org/10.5209/TEKN.64163

\title{
Resistencias sumergidas. Cartografía de la tecnopolítica feminista en México
}

Claudia Ivette Pedraza ${ }^{1}$; César Augusto Rodríguez Cano ${ }^{2}$

Recibido: 22 de abril 2019 / Aceptado: 7 de octubre 2019 Open peer reviews

Resumen. En este artículo se analizan las redes heterogéneas de actores clave implicados en los procesos de aprendizaje sobre el uso y apropiación de tecnologías digitales entre las mujeres. Mediante una propuesta teórica llamada tecnopolítica feminista, aunada a un abordaje metodológico que entrelaza el Análisis de Redes Sociales con la Teoría del Actor Red, se muestran los alcances y desafíos de las comunidades feministas para activar escenarios, herramientas y redes derivados de las tecnologías digitales.

Palabras clave: Análisis de Redes Sociales; ciberfeminismo; Tecnologías de la Información y la Comunicación; Teoría del Actor-Red.

\section{[en] Submerged resistances. Cartography of feminist technopolitics in Mexico}

\begin{abstract}
In this article we analyse the heterogeneous networks of actors who play a key role in learning processes about use and appropriation of digital technologies among women. Based on a theoretical proposal called 'feminist technopolitics' and a methodological approach where Social Network Analysis and Actor-Network Theory are intertwined, scopes and challenges for feminist communities to activate scenarios, tools and networks derived from digital technologies are highlighted.

Keywords: actor-network theory; cyberfeminism; Information and Communication Technologies; Social Network Analysis.
\end{abstract}

Sumario. 1. Introducción. 2. Marco teórico. 3. Metodología. 4. Resultados. 5. Conclusiones. 6. Referencias.

Cómo citar: Ivette Pedraza, C.; Rodríguez Cano, C. A. (2019). Resistencias sumergidas. Cartografía de la tecnopolítica feminista en México. Teknokultura. Revista de Cultura Digital y Movimientos Sociales, 16(2), 197-212.

\section{Introducción}

Durante los últimos años, el espacio público ha sido ocupado por numerosas manifestaciones feministas con el auxilio de herramientas en línea, lo cual representa

\footnotetext{
1 Universidad La Salle México (México)

E-mail: Claudia.pedraza@lasalle.mx

2 Universidad Nacional Autónoma de México (México)

E-mail: cesararcano@gmail.com
} 
un poderoso cambio en la lógica de los procesos de comunicación, organización y acción política tradicional.

Si bien históricamente este movimiento se ha articulado en forma reticular, con nodos y conexiones en diferentes niveles, lo que caracteriza esta nueva forma de levantamiento es la posibilidad de conectar los diferentes espacios, esfuerzos y recursos con una visibilidad inédita.

Las manifestaciones irrumpen de diversas formas: blogs, medios digitales, performances, instalaciones, producciones audiovisuales, consignas en hashtags que se vinculan con protestas, paros de labores, marchas, intervenciones arquitectónicas y un largo etcétera. En suma: acciones conectadas dentro y fuera del entorno digital, que han generado una narración pública y colectiva desde la experiencia de las mujeres. Como nunca antes, las tecnologías de la información y la comunicación han permitido transformar el silencio en lenguaje y acción, en el sentido que señalaba Lorde.

Baher (2016, p. 18) asegura que las plataformas digitales ofrecen un gran potencial para difundir ampliamente ideas feministas, nuevos modos de discurso sobre el género y el sexismo, y modos creativos de protesta, que se han convertido en hitos del movimiento feminista del nuevo siglo. En específico, el objetivo de este texto es analizar las redes heterogéneas de actores clave implicados en los procesos de aprendizaje sobre el uso y apropiación de tecnologías digitales entre las mujeres promovidas por los colectivos de tecnopolítica feminista, los cuales han acompañado las emergentes movilizaciones feministas.

Desde los primeros videos virales de las integrantes de Femen ${ }^{3}$ con el torso desnudo en 2008 hasta la gran conversación sobre el acoso y abuso sexual que generó el \#MeToo en más de 85 países en 2017, las acciones feministas en las redes sociodigitales han sido el punto de partida para movilizaciones transnacionales que difícilmente pueden ser ignoradas.

En América Latina esta lógica emergente también ha cobrado relevancia en el último lustro. Sancho (2018) ubica la primera manifestación articulada en las redes con el hashtag \#Niunamenos ${ }^{4}$ surgido en Argentina en 2015, que colocó la discusión del feminicidio como una emergencia compartida en la región. Para 2016, Ecuador fue el epicentro de otra manifestación por la defensa del derecho a la movilidad y a la seguridad bajo el hashtag \#ViajoSola ${ }^{5}$. En marzo de 2017, países como Argentina, Venezuela, Colombia y México se unieron a la convocatoria de la Primera Huelga Internacional de las Mujeres, un paro de labores convocado a través de las redes para denunciar la desigualdad, la discriminación y la violencia de género en los espacios laborales ${ }^{6}$.

En octubre de ese mismo año, la traducción del \#Metoo ${ }^{7}$ al \#Yotambién reveló historias de acoso sexual no solo en las industrias del entretenimiento, sino en todos

3 Femen es una organización feminista, ateo y abolicionista, fundado en Ucrania, caracterizada por sus protestas extremistas y transgresoras de diferentes espacios públicos.

$4 \quad$ La movilización surge como protesta ante el aumento de mujeres asesinadas en el país; el nombre primero se utiliza para un maratón de lectura y luego se convierte en consigna para convocar a una marcha celebrada en el mes de marzo.

5 Surgido tras el asesinato de las argentinas Marina Menegazzo y María José Coni, en la ciudad de Montañita, Ecuador, mientras estaban de excursión.

6 La Huelga Internacional de Mujeres, surgida en Polonia, convocó en su primera edición a cerca de 60 países. Para el 2018, se lograron conectar manifestaciones en más de 170 países.

7 Derivado de las múltiples denuncias contra las agresiones sexuales del productor de cine estadounidense Harvey Weinstein. 
los ámbitos sociales con participación femenina. Para 2018, la \#MareaVerde que representaba la lucha por la despenalización del aborto en Argentina se expandió por otros países latinoamericanos, que adoptaron el pañuelo, las consignas y la agenda de acciones políticas para garantizar el derecho de las mujeres a un aborto libre y seguro.

En todos los casos, de una situación individual y local surgió una movilización colectiva y supranacional, que hizo evidente el carácter estructural de los mecanismos de violencia de género que enfrentan en su vida cotidiana las mujeres de estos países.

En el caso de México, la emergencia de las movilizaciones feministas también estuvo provocada por la violencia de género (Inmujeres CDMX, 2016; INEGI, 2017). Desde 2013, el injusto encarcelamiento de Yakiri Rubio, una joven capitalina acusada de homicidio tras apuñalar en defensa propia al hombre que la violó, provocó una marcha de protesta convocada con los hashtags \#LIberenaYakiri \#LIbertaparaYaki y \#JusticiaparaYaki. Este hecho mostró la visibilidad que podían dar las redes, originando otras convocatorias para manifestarse por casos particulares, algunos con mayor respuesta que otros, en medio de un número creciente de feminicidios. El malestar ante la violencia eclosionó en abril del 2016 con la denominada Primavera Violeta \#24A, la primera manifestación realizada fuera de los días institucionalizados para la protesta feminista ${ }^{8}$. Con el nombre oficial de "Movilización Nacional contra las Violencias Machistas", derivada de casos de acoso y violencia sexual que cobraron una notable visibilidad ${ }^{9}$, mujeres de más de 40 ciudades salieron a las calles convocadas por la consigna \#VivasNosQueremos. A la par de la marcha, la Primavera Violeta originó una contundente narración colectiva en las redes socio-digitales bajo el hashtag \#Miprimeracoso, al que se sumaron miles de mujeres de otros países conectadas por experiencias compartidas de violaciones, abusos y agresiones sexuales.

La Primavera Violeta hizo evidente el potencial de las redes feministas articuladas en colectivos, organizaciones, medios alternativos, que desde ese momento no han dejado de manifestarse públicamente. En 2017, la consigna \#SiMeMatan reactivó la movilización en red para denunciar la criminalización de las víctimas de violencia de género por parte de autoridades y medios de comunicación ${ }^{10}$. El incremento de asesinatos de niñas y mujeres durante 2018 hizo que el hashtag \#FeminicidioEmergenciaNacional fuera tendencia en diferentes momentos, acompañado de marchas, infografías, videos, presentación de informes e investigaciones realizadas por páginas web, medios feministas y organizaciones que hicieron común el objetivo de denunciar la violencia de género. El 2019 inició con dos nuevas demostraciones de la efectividad para organizar y visibilizar a la creciente multitud feminista. Primero, con una acto individual que a la vez fue multitudinario: la protesta en redes para reivindicar el derecho a transitar por espacio público en condiciones de seguridad, que originó la

$8 \quad$ El Día Internacional de la Mujer, 8 de marzo; El Día Internacional Para la Erradicación de la Violencia contra las Mujeres, 25 de noviembre.

9 La violación múltiple de Daphne Fernández en Veracruz y el acoso sexual a la periodista Andrea Noel en la ciudad de México.

10 La movilización surgió tras el asesinato de Lesvy Berlin, estudiante de la Universidad Nacional Autónoma de México, en las instalaciones de la institución. Meses más tarde, el mismo hashtag convocó a una segunda movilización por un nuevo caso: el asesinato de Mara Castilla, en Puebla, ejecutado por un conductor de Uber. 
producción de miles de fotografías y videos bajo el lema \#LaCalleEsNuestra ${ }^{11}$. Una semana más tarde, en medio de una ola de denuncias por secuestros de mujeres en el sistema de transporte público de la Ciudad de México, se convocó a otra marcha que recuperó la consigna \#Vivasnosqueremos surgida en la Primavera Violeta. Esta vez, la movilización estuvo acompañada de otras acciones colectivas, espontáneas o planeadas, organizadas en red y enlazadas con otros espacios fuera de línea.

Aunado a esto, además del potencial movilizador de las redes socio-digitales, lo que estas manifestaciones revelaron fue la existencia de una red de colectivos feministas que, en conexión con diferentes actores (no necesariamente feministas), han estructurado procesos permanentes para fomentar el aprendizaje en torno al uso, apropiación y transformación de la propia tecnología. Estos colectivos se distinguen no solo por utilizar las tecnologías para organización y comunicación de movilizaciones o acciones específicas, las cuales han monopolizado el análisis social, sino que tienen por fin involucrar de manera menos visible pero más constante a otras mujeres, a través de cursos especializados sobre software libre, programación, producción audiovisual, estrategias de seguridad digital, etc., así como de la enseñanza de los repertorios de protesta, entre otros recursos.

A pesar de que el tema que conecta a esta red de colectivos es el de género y tecnologías, no están desvinculados de otras causas del movimiento feminista: la violencia de género, la salud reproductiva, la igualdad política, la defensa de la tierra, la educación, el acceso al trabajo, etc. En este texto, se propone analizar dicha red de colectivos como una irrupción sumergida a partir del término tecnopolítica feminista.

\section{Marco teórico}

A lo largo de más de cuatro décadas, diferentes autoras han problematizado la relación de las mujeres con las tecnologías desde la categoría de género (Keller, 1985; Cockburn y Ormrod, 1993; Harding, 1996). En este sentido resalta el cuestionamiento a la supuesta neutralidad de la ciencia y la tecnología, la cual oculta que ambas actividades reproducen las desigualdades de género existentes en la sociedad. Lo anterior se manifiesta en diversas dimensiones del ámbito científico: las brechas numéricas en las profesiones científicas y tecnológicas, la poca visibilidad de la participación de las mujeres en la generación de conocimiento, la desvalorización de los conocimientos y las tecnologías que se consideran femeninos, la jerarquía de los tipos de desarrollos científicos y tecnológicos supuestos prioritarios, la validación de las formas de producir conocimiento científico y tecnológico.

No obstante, desde la perspectiva feminista también se ha reconocido el potencial subversivo de las tecnologías, implícito en los usos, apropiaciones y re-significaciones que con ellas realizan las mujeres.

Este potencial subversivo se hizo más evidente con el uso de las tecnologías digitales. Apropiándoselas, las mujeres han podido visibilizar problemáticas, reivindicar derechos y organizar acciones para desafiar el orden de género, articulando un movimiento en, por y para esta red: el movimiento ciberfeminista. Gran parte del

11 Convocada tras la propuesta de ordenar un toque de queda para la población femenina realizada por la diputada veracruzana Miryam Ferraez, a fin de evitar los feminicidios. 
movimiento se ha centrado en explorar las posibilidades que ofrecen estas tecnologías en los dos primeros sentidos: como escenario (en) y como herramienta (por) para la transformación, a través de procesos de denuncia, creación y producción de información. Instalaciones artísticas, blogs y páginas web, producciones audiovisuales, fanzines o medios alternativos son manifestaciones de estas posibilidades. Pero en los últimos años, la irrupción de múltiples movilizaciones feminista de carácter transnacional y que están conectadas a través de la red, ha permitido constatar el tercer sentido: el de la lógica reticular, que encadena espacios, actores, mensajes y dispositivos. Esta irrupción, a su vez, acarrea la necesidad de enfoques innovadores para mirar la relación de las mujeres y las tecnologías digitales.

Algunos trabajos sobre el movimiento ciberfeminista señalan que dicha relación se ha abordado desde dos grandes posturas: la utópica, sustentada en el planteamiento original del ciberfeminismo de Plant; y la distópica, representadas por la propuesta tecnofeminista de Wajcman (Boix, 2015; Reverter Bañón, 2013; Braidotti, 2004). Estas posturas no son solamente analíticas (es decir, una forma de explicar las relaciones entre mujeres y tecnologías digitales) sino también políticas (una propuesta de construir las relaciones entre mujeres y tecnologías digitales para transformar las relaciones de poder).

Plant (1998) concibe el ciberfeminismo como una insurrección (material, económica, social) hecha de enlaces entre mujeres, computadoras, comunicaciones, todo en conexión en el ciberespacio (imaginado como un espacio libre de cualquier construcción genérica). Para Plant, la estructura fluida, relacional, horizontal y rizomática de Internet es similar a las formas de acción y organización caracterizadas como femeninas, por lo cual esta red resulta idónea para subvertir el orden de género: las mujeres pueden adaptar, rediseñar y recodificar las tecnologías, desmontando en sus usos la diferencia de poder implícita en la dicotomía social de masculino/femenino (así como otras sustentadas en la misma dualidad: cuerpo/mente, biología/tecnología, natural/artificial, etc.). Para esto, el ciberfeminismo apuesta por la propuesta política de producción de identidades que reivindican lo femenino, o en sus intentos más radicales, estar fuera de la dicotomía genérica (como la identidad Cyborg, de Haraway). Al adaptar, rediseñar y recodificar las tecnologías, las mujeres hacen lo mismo con su identidad: a través de producción de mensajes, instalaciones artísticas o intervenciones, ellas deciden qué quieren ser y cómo quieren definirse.

En contraparte, la propuesta tecnofeminista de Wajcman (2004) plantea que Internet y las tecnologías digitales sostienen la estructura patriarcal de las sociedades por lo cual, más que transformar y liberar, acentúan la dominación de las mujeres. Para Wajcman (pp.15-17), las relaciones de género y tecnología, se co-construyen: de origen, existe un marco de valores atravesados por la dicotomía masculinidad/ feminidad que determinan el sistema de producción científica y tecnológica. Por un lado, esto origina un proceso de socialización diferenciada de hombres y mujeres en el uso de las tecnologías; por el otro, existe un sesgo en el diseño y producción de los artefactos, las prácticas, los usos y los significados socioculturales que se consideran válidos para la tecnología, lo cual impide que todo aquello caracterizado como $f e$ menino tenga el mismo valor. En función de esta doble exclusión de lo femenino, el tecnofeminismo cuestiona el sistema de valores que sustentan y son sustentados por las tecnologías, señalando que éste no desaparece por las producciones de identidad que a través de ellas realizan las mujeres. Por eso, la propuesta política es incorporar a la población femenina no solo al uso sino a la producción de tecnología y de 
conocimientos tecnológicos, para subvertir y redefinir su marco de valores (Zafra Alcaraz, 2004; Wajcman 2004; Baidotti, 2002).

Aunque estas dos propuestas definen líneas de análisis y de acción muy específicas, el movimiento ciberfeminista se manifiesta en actos que van desde la subversión de la identidad femenina a través de un performance interactivo hasta modificaciones de dispositivos, programas e infraestructura tecnológica a través de colectivas hackfeministas. Por esta razón, algunas autoras (Boix, 2015; Núñez Puente, 2011; Reverter Bañón, 2013; Rovira Sancho, 2018) señalan que ambas posturas simplifican la compleja relación entre mujeres y tecnología que existe en dicho movimiento:

La mirada a las tecnologías no puede quedarse en una mera observación radicalizada en un sentido de euforia por un lado o de pesadumbre por otro. Tanto el discurso utópico o tecnofílico, como el distópico o tecnofóbico suponen, además, análisis desajustados respecto a los planteamientos críticos que las feministas han venido haciendo desde hace décadas en el terreno de la ciencia y las tecnologías (Reverter, 2013, p. 453).

La pregunta que surge entonces es: ¿cómo recuperar el potencial subversivo presente en la diversidad de prácticas que se realizan dentro del movimiento ciberfeminista sin idealizar ni desacreditar a las tecnologías digitales que lo articulan? Para responder a esto, se coincide con Boix (2015), quien plantea la necesidad de transitar hacia una tecnopolitica feminista (originalmente nombrada por ella misma como "ciberfeminismo social") como una propuesta para analizar el actual devenir del movimiento. Esta propuesta recupera el potencial transformador de las tecnologías que reconoce el ciberfeminismo y la necesidad de transformar las estructuras patriarcales presentes en los ámbitos tecnológicos que señala el tecnofeminismo; pero además, coloca la estructura y dinámica en red como la clave para analizar y construir estos procesos de transformación.

Toret (2013) define tecnopolítica como un patrón de autocomunicación, auto organización y acción de la sociedad en red, que apela a principios colaborativos, a la propagación de ideas a través de plataformas digitales y la sincronización de afectos para la consecución de objetivos colectivos y conectivos. En la misma línea, Sierra y Gravante hablan de tecnopolítica como una mediación transformadora y descentralizada originada por las tecnologías digitales, basada en la democracia del código y la creación colectiva (2017, p. 7). Lo que distingue a este patrón (que define una estructura) o a esta mediación (que define una dinámica) es el aspecto de la reticularidad, es decir, la estructura y la dinámica en red como resultado de enlaces cruzados en todos los niveles, donde las actividades difieren entre colectivos pero se desarrollan conjuntamente a partir de objetivos compartidos.

Treré y Barranquero (2017) entienden tecnopolítica a partir de una concepción profunda, compleja y no instrumental de la relación entre política y tecnología, la cual refiere la redefinición de los espacios, el tiempo y los procesos de la política a causa de las tecnologías de la comunicación . Desde esta misma perspectiva, Sierra (2018, pp. 985-987) retoma la idea de un espacio público oposicional (Negt, 2007), cuyas líneas prioritarias de los procesos emergentes de comunicación en los frentes culturales que acompañan el activismo tecnopolítico son: 1) la apropiación tecnológica y organización social, es decir los modos propios de comunicación, las estéticas de resistencia, los contornos del espacio público antagonista en sus formas de expresión y autogestión; 2) interactividad y nuevos modos de mediación social, en particular la necesidad de discutir en detalle los niveles y formas de interacción; 
3) memoria y gestión local del conocimiento, o sea de configuración democrática y política de las exomemorias digitales y la capacidad de autonomía y autodeterminación de las culturas populares, y 4) economía política del cambio tecnológico y la innovación social, donde es preciso politizar críticamente la generación social de la comunicación y la cultura en una época de creciente disgregación y mercantilización del universo simbólico.

Es decir, los procesos emergentes de comunicación en el espacio público oposicional indican prácticas de apropiación tecnopolítica: cuestionamiento, organización, resistencia, expresión y autogestión, con el añadido de que pueden ser tanto manifiestas como sumergidas como se expone desde la teoría de los nuevos movimientos sociales (Melucci, 1999). Un planteamiento que nos recuerda la noción de contrapúblicos subalternos de Fraser (1990) para definir arenas discursivas paralelas donde los miembros de los grupos sociales subordinados inventan y circulan contradiscursos que les permiten formular interpretaciones oposicionales de sus identidades, necesidades e intereses.

En función de estos planteamientos, nos preguntamos iqué implica hablar de una tecnopolítica feminista? En primer lugar, una propuesta analítica del movimiento ciberfeminista en torno a la estructura y dinámica de red que se articula con otros participantes; y en segundo lugar, una propuesta política, a la manera de Rancière que entiende lo político como la parte de los que no tienen parte (1996), para que la conexión entre mujeres, tecnologías y otros actores transforme las relaciones de poder, como el objetivo común compartido por esa red. Ambas propuestas, atravesadas por el principio feminista de colocar en el centro la experiencia de las mujeres.

Con dicho principio, la tecnopolítica feminista revalora la relación mujeres-tecnologías en los múltiples espacios en los que ocurre, no solo con las tecnologías digitales sino con otras tecnologías de la reproducción, el cuidado, la alimentación, la creación artística; es decir, recuperando la dimensión política de la vida cotidiana. Reverter (2001, p. 48) señala que es necesario integrar todas estas experiencias tecnológicas de las mujeres en un sistema que intente explicar las formas de subordinación e insubordinación ante las tecnologías. En este proceso se relaciona el espacio público con el espacio privado, la dimensión biográfica individual y comunitaria, las necesidades cotidianas y las necesidades estructurales (Núñez Puente, 2011, p. 88) a través de usos y acciones tácticos que van de lo racional a lo afectivo, de la visibilidad a la latencia y de la organización a la acción.

Se entiende que el uso, apropiación y resignificación de las tecnologías se da en un marco de conflictos y contradicciones, en el cual también están los intereses de las corporaciones tecnológicas, la economía de la información, la mercantilización de los datos, el capitalismo cognitivo, etc., pero que no desacredita la distribución de representaciones políticas en un marco general de la vida social.

\section{Metodología}

Desde su origen, el análisis de los fenómenos tecnopolíticos ha traído consigo innovaciones y desafíos metodológicos. Al involucrar escenarios inéditos en cuanto a infraestructura socio-técnica, junto con fenómenos culturales complejos, los abordajes han tendido a ser multidisciplinarios (Congosto y Laniado, 2013; Aguilera, 2013). 
Es por eso que el enfoque de análisis de este artículo se atreve a continuar con la tradición de la experimentación metodológica al entretejer dos enfoques de investigación: en primer lugar, el Análisis de Redes Sociales (ARS) y, en segundo lugar, una aproximación desde la Teoría del Actor-Red (TA-R) ${ }^{12}$.

Como sabemos, el ARS ha sido una técnica de amplio bagaje desde diferentes enfoques científicos, entre ellos las matemáticas, la psicología y la sociología. Desde antes de la emergencia de Internet y su arquitectura reticular, las formaciones sociales se han estudiado entendiendo a los actores como nodos y sus relaciones como vínculos (véase por ejemplo el clásico estudio de Granovetter, 1983). En términos estrictamente cuantitativos, el Análisis de Redes Sociales es un enfoque estadístico que tiene por intención medir diferentes aspectos relacionales de las redes que se estudian.

La TA-R se dedica también al estudio de las redes sociales aunque pretende ir más allá. Como apunta Latour (2008), este enfoque supone una ontología del actante-rizoma, en la cual los actores son heterogéneos y las redes se crean a partir de las mediaciones de los actores no de una manera técnica sino social en sentido diverso y ramificado. De acuerdo con este postulado, se debe trascender la sociología entendida como ciencia de lo social para enfocarse en una sociología de las asociaciones, un giro epistemológico en pos del reensamblado social; desde este punto de vista, la noción de actante es fundamental para comprender la heterogeneidad de los actores involucrados pues alude tanto a nodos humanos como no humanos, aspecto fundamental en el contexto de las tecnologías digitales ${ }^{13}$.

En síntesis, el entretejido de la propuesta latouriana con la dimensión topológica de las estructuras en red vislumbra un acercamiento más panorámico del fenómeno de estudio que aquí analizamos, al entender la conformación de redes a manera de mapas mientras que el análisis de los mismos como un ejercicio cartográfico.

Con este planteamiento, la hipótesis central es que dentro del movimiento tecnopolítico feminista en México existe una confluencia de redes heterogéneas que han definido las agendas más recientes del espacio público oposicional. Es decir, hay colectivos y activistas, a la usanza tradicional de los estudios que entienden redes a partir de las cuentas de usuario. Pero también emergen otros nodos entrelazados, de diferente índole, que definen esta irrupción en planos heterogéneos: materiales, ideológicos, tecnológicos, geográficos, etcétera.

Con este fin se recolectó una base de datos a partir de un cuerpo de investigación muy específico -los carteles de difusión de eventos-, como veremos más adelante. Contra la tendencia de sistematizar grandes cantidades de información bajo la perspectiva de grandes datos -big data- que arrojan los corpus contemporáneos, muchas veces en riesgo de centralizar y/o extraviar el propio análisis (Boyd y Crawford, 2012), se decidió realizar una detallada recopilación de nodos y vínculos de las formas de organización tecnopolítica feminista con la ventaja de razonar a conveniencia teórica de la investigación, un enfoque entendido desde la perspectiva de los datos densos (Wang, 2013) y el ethos descriptivo de la TA-R. Para ello se siguieron

12 El trabajo metodológico que dio origen a este artículo partió del proyecto de investigación titulado "Internet y democracia en la transformación estructural de la vida pública: Análisis de Redes Sociales y fenómenos tecnopolíticos en México", registrado el año 2018 en la Universidad Autónoma Metropolitana unidad Cuajimalpa, de la Ciudad de México.

13 Un referente en la aplicación de la Teoría del Actor-Red es el trabajo de Magallanes-Blanco y RodríguezMedina (2016). 
varios pasos. En primer lugar, se determinaron los colectivos feministas más visibles en México dedicados a promover exclusivamente procesos de aprendizaje sobre el uso y apropiación de tecnologías entre las mujeres, de lo cual destacaron Ciberseguras, Luchadoras y Laboratorio de Interconectividades ${ }^{14}$ (Internet Governance Forum, 2017). Después se exploraron sus cuentas de redes sociales para elegir en qué plataforma tenían mayor actividad dedicada a difusión. Por ello se retomaron de sus espacios en Facebook los eventos en los que estuvieran involucrados estos colectivos en el plazo del 24 de abril de 2016 al 25 de noviembre de 2018. Esta selección obedeció a que el 24 de abril de 2016 fue la primera movilización convocada por redes sociodigitales que no fue organizada alrededor de una fecha específica, sino en atención al contexto de violencia (acompañada por las etiquetas \#24A, \#PrimaveraVioleta) mientras que el 25 de noviembre de 2018 fue el Día Internacional de la Violencia contra la Mujer más reciente, la cual es en sí una fecha significativa pero también un cierre que concedió más de dos años y medio de eventos tecnopolítico-feministas en México, una selección suficiente para los objetivos de este estudio. De tal forma, a partir de los carteles (ver Imagen 1) de la difusión de 82 eventos realizada por los propios colectivos, luego de una primera fase de reflexión y análisis sobre los datos presentes en estas publicaciones, se recolectó la información relacional a partir de las siguientes categorías: colectivos $^{15}$, expositores, temáticas, sedes, ubicaciones, modalidades y respaldos ${ }^{16}$.

Imagen 1. Ejemplo de cartel seleccionado. Fuente: Elaboración propia

\section{Ciberseguras se suma desde México al FEMHACK: \\ Adas conectAdas: Reconstruyendo la Red en Mesoamérica}

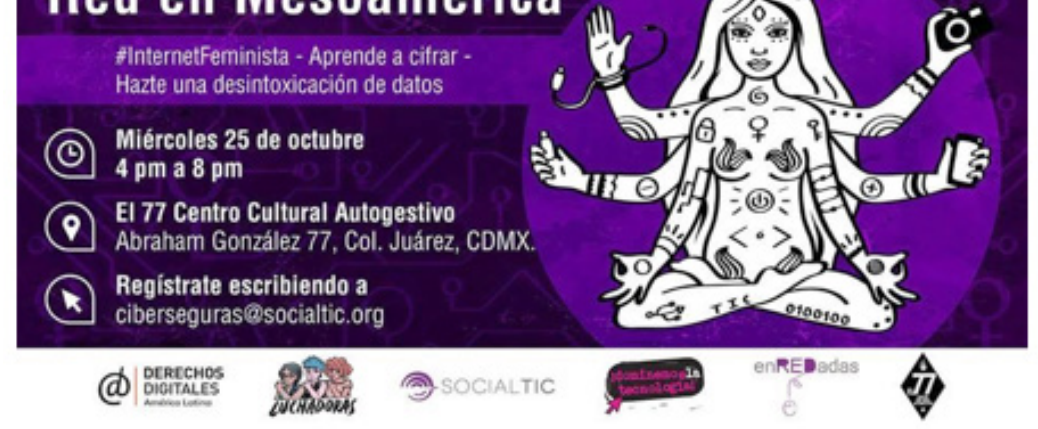

14 Ciberseguras es una iniciativa surgida originalmente en México, actualmente con la participación de colectivos de Brasil, Bolivia y Guatemala, que comparten recomendaciones y recursos de autodefensa digital a través de talleres y materiales en la web; Luchadoras se dedica a la producción de información con perspectiva de género (a través de su sitio web) así como a la realización de talleres sobre TICs y Género; y el Laboratorio de Interconectividades, propuesta hackfeminista que trabaja en conjunto con MedialLabMx y Comando Colibrí.

15 Los colectivos feministas se autodenominan "colectivas" como una táctica para distinguirse de otras organizaciones sociales. En la revisión de los carteles también se encontraron colectivos no feministas dentro de esta categoría.

16 Solamente se tomaron en cuenta los carteles publicados por las propias cuentas de los Colectivos, aun cuando se tiene la noción de su participación en otras actividades que no fueron difundidas por esta vía. 
El análisis estadístico consistió en la aplicación de las instrucciones modularidad y centralidad de grado, ambas representadas visualmente mediante la instrucción de espacialidad Force Atlas, la primera para poner en funcionamiento el algoritmo de detección de límites comunitarios por la fuerza entre sus vínculos y la segunda para ilustrar el número de menciones de un nodo, por diferencia de colores y de tamaños respectivamente -de diferente color, diferente comunidad; a mayor tamaño, mayor centralidad (ver figura. 1) ${ }^{17}$.

Figura 1. Visualización. Fuente: Elaboración propia

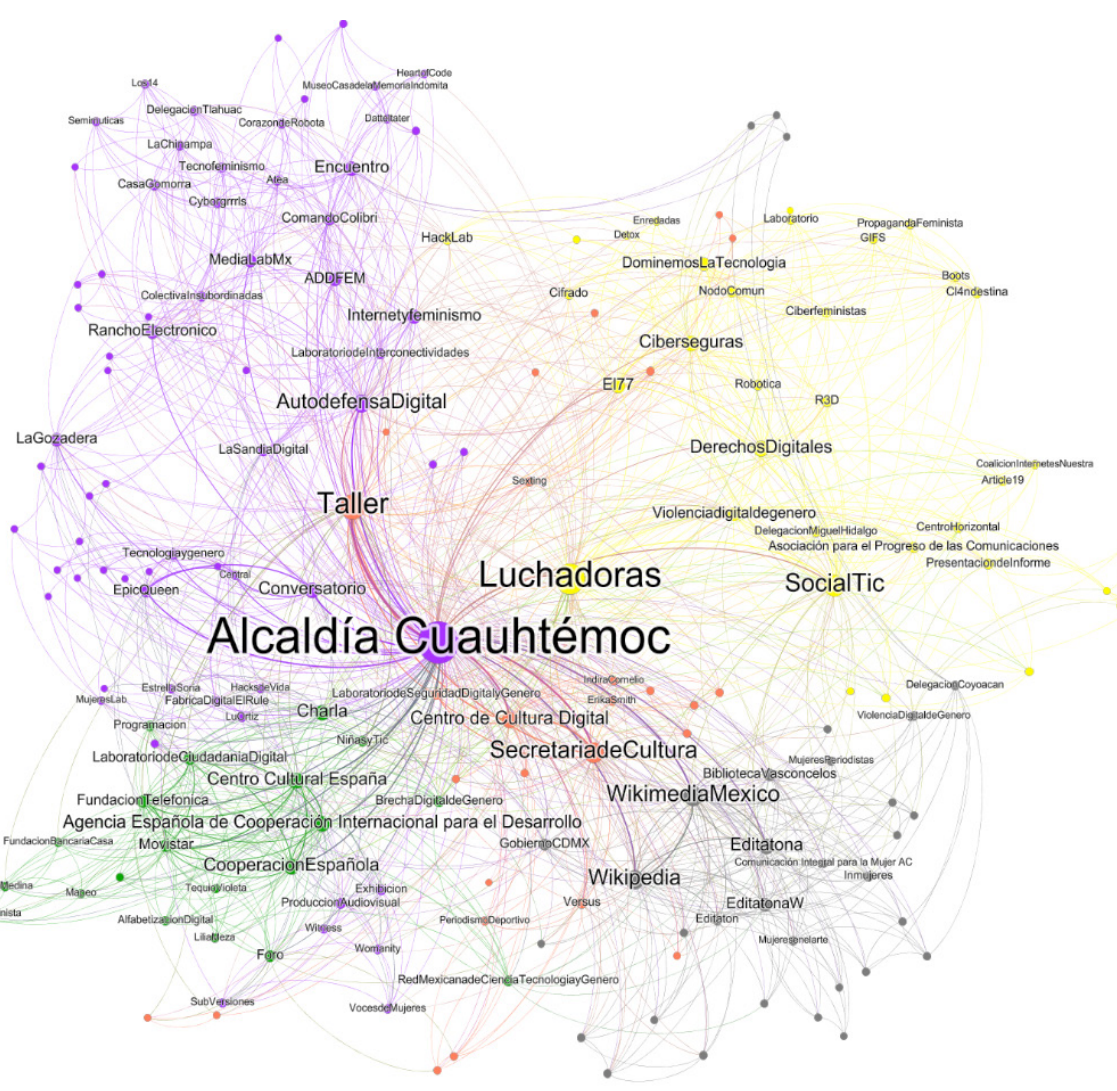

La interpretación y los resultados se realizaron bajo una perspectiva híbrida, basados en una descripción con base en la visualización de las comunidades y los grados.

17 Los nodos con etiqueta tienen una centralidad de grado igual o mayor a 10; es decir, igual o mayor a diez menciones. Los nodos sin etiquetas tienen un grado menor a diez. Este recurso se utilizó para facilitar la lectura de la totalidad del grafo. En cuanto a los resultados estadísticos, la red tiene un diámetro de 7, un grado medio de 7.885 y un índice de modularidad de 0.388 . 


\section{Resultados}

En los registros por categorías se distingue 50 temáticas, 37 respaldos, 32 colectivos ( 25 colectivos que trabajan el tema de tecnologías, 7 que trabajan otros temas), 19 sedes ubicadas en 9 localidades, 14 expositores y 14 modalidades. Al observar la red, se pueden distinguir cuatro comunidades con enlaces colaborativos bien definidos.

En primer lugar con un 36\% de la red está la comunidad que se enlaza a través del tema Autodefensa Digital Feminista, principalmente con los colectivos MediaLabMx, ADDFEM, Comando Colibrí y la Sandía Digital (con quienes también trabajan el tema de Internet y Feminismo). Esta comunidad (representada en color violeta) conecta, a través de colaboraciones múltiples, a otras colectivas y espacios con menos notoriedad en la red. Algunos eventos son promovidos por las sedes, particularmente por los centros comunitarios como Rancho Electrónico, La Gozadera, E1 77 o la Fábrica Digital El Rule. Otras surgen de colectivos como Insubordinadas, Hacks de Vida o Corazón de Robota. Las principales modalidades de las prácticas de esta comunidad son Talleres, Conversatorios y Encuentros (como el de Cyborg Grrls, que se realiza cada año en el mes de marzo). Dentro de los Encuentros se registraron vínculos con respaldos internacionales como Internet Society México, Internet Society Woman, Dattellater, o Heart of Code, que apoyan el desarrollo de iniciativas tecno y ciberfeministas. Otro rasgo distintivo de esta comunidad es el trabajo periférico: son quienes expanden la red más allá de la ubicación central de la Alcaldía Cuauhtémoc, con acciones en puntos como Milpa Alta, Tláhuac, Ecatepec, entro otros.

La segunda comunidad, en color amarillo, con un 19\% de la red, se conecta a través de los colectivos Luchadoras y Ciberseguras, en alianzas con organizaciones nacionales e internacionales como Social Tic, Artículo 19, APC, Dominemos la Tecnología o Derechos Digitales, los cuales trabajan en torno a la generación de procesos de ciudadanía (no solo de las mujeres) a través de las tecnologías de la información y la comunicación. Particularmente, por vía del proyecto Ciberseguras, esta comunidad se enfoca en el tema de Violencia Digital de Género, predominantemente con las modalidades Taller y Conversatorios. En estos, la finalidad es reflexionar sobre las tecnologías, reconocer las formas de violencia y promover el aprendizaje de estrategias de autodefensa en línea (sobre todo a través de prácticas de cifrado). Por este mismo proyecto, la comunidad se conecta con colectivos e iniciativas feministas de otros países de América Latina, como Nodo Común (Bolivia), Ciberfeministas (Guatemala) o Cl4andestina (Brasil). Destaca la presentación del Informe "Violencia en línea contra las mujeres en México", un trabajo en el cual se crea una tipología de las modalidades de violencia digital de género, el único en su clase registrado en la categoría modalidades.

Si bien conectadas por diferentes nodos, los tres colectivos de los que parte el análisis aparecen con la evidencia de sus cercanías en estas dos primeras comunidades, en un tono de organizaciones sociales. En tanto, las dos comunidades restantes claramente aparecen vinculadas con una intervención institucional.

Así, se distingue en color verde la tercera comunidad tiene como nodo articulador el Centro Cultural España (CCE) como sede, principalmente a través del proyecto Laboratorio de Ciudadanía Digital y su estructura de difusión. Por su origen, el CCE cuenta con el respaldo institucional de la Agencia Española de Cooperación Internacional para el Desarrollo, de Cooperación Española, así como de Fundación Telefónica y la compañía Movistar, ambas de la iniciativa privada, que apoyan con la 
gestión de recursos económicos. En el CCE, colectivos presentes en las otras comunidades - como Luchadoras, Hacks de Vida, Mujeres Lab o Subversiones- realizan actividades en diversas modalidades: Taller, Foro, Exhibición y Panel, desde la óptica de la alfabetización y educación digital. No obstante, en esta comunidad sobresale la modalidad Charla y, por ende, hace más visible la categoría Expositores, es decir, expertos y expertas invitados a compartir sus conocimientos. Los temas abordados presentan menos centralidad, pero una mayor diversidad: Programación, Hackeo, Producción Audiovisual, Robótica, entre otras.

La última comunidad surge en torno al trabajo de Wikimedia México, que muestra en color gris. En razón de esto, la modalidad predominante es la Editatona, definida como una jornada de edición de artículos de Wikipedia sobre mujeres destacadas o temas de género, en las cuales solo participan mujeres como editoras. Las Editatonas se realizan en torno a temas únicos: Mujeres y Política, Mujeres y Ciencia, Mujeres y Arte, Mujeres Futbolistas, Derechos Políticos, Interrupción del Embarazo. Debido a las temáticas variadas, en esta red se presenta la mayor cantidad de colectivos y organizaciones que no trabajan el tema de las tecnologías pero que apoyan con la gestión de información sobre las jornadas de edición, por ejemplo: Versus (deportes), La Red Mexiteg (ciencia y tecnología), CIMAC (periodismo) o ANDAR (interrupción segura del embarazo). Esta comunidad se asocia con los respaldos de organizaciones como la propia Wikipedia, Wikimedia Ibero Coop o WikiWoman, todas vinculadas al trabajo de la enciclopedia colectiva. También ha generado alianzas con instituciones gubernamentales, visualizadas en un cluster muy cercano en color anaranjado, como la Secretaría de Cultura, el Gobierno de la Ciudad de México y el Instituto Nacional de las Mujeres (Inmujeres), las cuales facilitan Sedes como el Centro de Cultura Digital (uno de los nodos más fuertes de la red), la Biblioteca Vasconcelos, el Museo Universitario de Arte Contemporáneo (MUAC), el Museo del Chopo, entre otros.

En cuanto a la centralidad de grado, es decir la importancia de los nodos en la red por el número de menciones, aparece claramente la Alcaldía Cuauhtémoc al centro, lo cual en sí es una redundancia pues esta demarcación representa el centro de la Ciudad de México y del país entero. De las 19 sedes detectadas, 13 se ubican en esta zona, lo cual indica una profunda centralización del movimiento tecnopolítico feminista, que enmarca la posibilidad de extender la red.

Enseguida aparece Luchadoras, confirmando su importancia en esta red, seguida de la modalidad Taller, la organización SocialTIC, la Secretaría de Cultura de la Ciudad de México, Wikimedia México, Wikipedia, el tema Autodefensa Digital, el Centro de Cultura Digital y la Cooperación Española.

Los nodos de la categoría Expositores son los que se presentan con menor fuerza, aunque los nombres que aparecen se identifican como parte de los Colectivos (Indira Cornelio, de Ciberseguras; Lili Anaz, del Laboratorio de Interconectividades) o de los Respaldos (Erika Smith, de APC; Nidia Chávez, de Telefónica).

Esto muestra que no solo los colectivos determinan la configuración de la red, sino que otros actantes como Temáticas, Modalidades, Respaldos y Sedes generan vínculos decisivos para el objetivo común: promover procesos de aprendizaje sobre el uso y apropiación de tecnologías entre las mujeres. Al buscar intencionalmente una red heterogénea en el sentido de la TA-R, el resultado es una interesante asociación de actores y actantes que ilustran la perspectiva sociológica de la tecnopolítica feminista en México (ver cuadro 1). 
Cuadro 1. Principales nodos por categoría. Fuente: Elaboración propia.

\begin{tabular}{|l|l|}
\hline \multicolumn{2}{|c|}{ Principales nodos por categoría } \\
\hline Colectivos & $\begin{array}{l}\text { Luchadoras, Social TIC, Wikimedia México, Ciberseguras, Derechos Digita- } \\
\text { les, Dominemos la Tecnología, MediaLabMx, ADDFEM, Epic Queen y Co- } \\
\text { mando Colibrí. }\end{array}$ \\
\hline Expositores & $\begin{array}{l}\text { Estrella Soria, Lu Ortiz, Erika Smith, Edith Medina, Indira Cornelio, Lilia } \\
\text { Meza, Andonella, Felipe García, Plaqueta y Nidia Chávez. }\end{array}$ \\
\hline Temáticas & $\begin{array}{l}\text { Autodefensa digital, internet y feminismo, violencia digital de género, brecha } \\
\text { digital de género, cifrado, tecnología y género, tecnofeminismo, robótica, pro- } \\
\text { ducción audiovisual y programación. }\end{array}$ \\
\hline Sedes & $\begin{array}{l}\text { Centro de Cultura Digital, Centro Cultural España, El 77, Rancho Electrónico, } \\
\text { La Gozadera, Biblioteca Vasconcelos, Casa Gomorra, Fábrica Digital El Rule, } \\
\text { La Chinampa y Centro Horizontal. }\end{array}$ \\
\hline Ubicaciones & $\begin{array}{l}\text { Alcaldías Cuauhtémoc, Tláhuac, Miguel Hidalgo, Coyoacán, Benito Juárez y } \\
\text { Milpa Alta, así como los estados de Jalisco, México y Puebla. }\end{array}$ \\
\hline Modalidades & $\begin{array}{l}\text { Taller, conversatorio, editatona, charla, encuentro, foro, hacklab, presentación } \\
\text { de informe, editatón y exhibición. }\end{array}$ \\
\hline Respaldos & $\begin{array}{l}\text { Secretaría de Cultura, Wikipedia, Cooperación Española, Agencia Española de } \\
\text { Cooperación Internacional para el Desarrollo, Editatona W, Movistar, Funda- } \\
\text { ción Telefónica, Laboratorio de Ciudadanía Digital, Gobierno de la Ciudad de } \\
\text { México y Asociación para el Progreso de las Comunidades. }\end{array}$ \\
\hline
\end{tabular}

Metodológicamente, el vínculo entre el ARS y la TA-R dio como resultado un ejercicio cartográfico inédito, en el que se vislumbra la heterogeneidad de la escena tecnopolítica feminista en México, con sus alcances y limitaciones ilustrados por categoría. Como se observa en el cuadro, los temas de interés versan tanto en el sentido del ciberfeminismo como del tecnofeminismo. Sin embargo, como planteamos en el plano teórico, lo que importa no es la distinción entre estas dos visiones sino la inmensa gama de posibilidades que existen entre ellas.

En cuanto a las categorías propuestas para realizar el mapeo, destacó la centralidad y centralización del movimiento en el corazón geográfico de la Ciudad de México, lo que hace evidente la necesidad de una descentralización de los esfuerzos tanto por parte de los colectivos como de las instituciones involucradas. En este sentido, para combatir la brecha cognitiva y pese a sus limitaciones, los espacios comunitarios de otras localidades surgen como alternativa a las sedes institucionales.

Dentro del ámbito didáctico, destaca la centralidad del Taller, una modalidad que pone en el centro de la tecnopolítica la práctica en el proceso de aprendizaje, realizado además de forma colectiva. Aunque esta modalidad es predominante, los formatos Conversatorio, Charla y Encuentro indican que también se busca un proceso dialógico de las experiencias de las mujeres.

En la categoría Temáticas, la Autodefensa Digital es central, lo que confirma la propuesta tecnopolítica feminista de promover aprendizajes que reconozcan la capacidad de acción de las mujeres para resolver los problemas apremiantes de su entorno. Particularmente, dado el contexto de violencia de género (digital y fuera de línea), dotar de herramientas y tácticas para enfrentarla a través de las tecnologías resulta fundamental para garantizar su participación pública. 


\section{Conclusiones}

Las irrupciones feministas de los últimos años en los espacios digitales han estado acompañadas por una vanguardia latente de tecnopolítica feminista basada en la activación pedagógica de redes heterogéneas.

En términos generales se encuentran tres fuerzas organizacionales de esta escena: los propios colectivos feministas, que destacan por sus alianzas con otros colectivos y organizaciones no feministas; las fundaciones internacionales, que se convierten en las principales fuentes de recursos; y el Gobierno de la ciudad, que aparece como un importante gestor para las Sedes.

Más que una tensión entre las tres fuerzas organizacionales encontradas, lo que se reconoce en dicha heterogeneidad es el aporte de las múltiples voces, proyectos y enfoques que integran y se vinculan en esta escena, lo cual se traduce en posibilidades de articular nuevos nodos, con prácticas, temas y actores que si bien no son evidentes, expanden y diversifican el espacio público oposicional. Esto es, con las temáticas, modalidades, colectivos y sedes conectados en la red se tejen procesos de apropiación tecnológica de las mujeres que superan el propósito de aprender a usar las tecnologías para dar paso a prácticas de organización, expresión, cuestionamiento y resistencia.

Así, en esta red no solo se mantiene el principio colaborativo y la reticularidad, sino además, emerge una propuesta de transformación de las relaciones de poder, en el amplio sentido tecnopolítico (y de las relaciones de poder de género, en el sentido feminista), y con ello la compleja renovación del espacio público oposicional.

Si bien los colectivos feministas visibles como nodos destacados de cada comunidad colocan la mediación transformadora originada por las tecnologías como objetivo común de la red, los otros agentes permiten diversificar los ámbitos en los que esta mediación puede adquirir su dimensión política: desde actividades públicas como el periodismo o el activismo, hasta las prácticas digitales personales cotidianas.

No obstante, se reconoce que la centralidad geográfica e institucional de algunos agentes aparece como un elemento limitante de esta expansión y diversificación, además de suponer riesgo de institucionalización, dependencia o privatización de la escena tecnopolítica feminista. El posible contrapeso de esta centralidad se encuentra en los numerosos nodos menos visibles, a manera de contrapúblicos reticulares, que proponen colaboraciones, temáticas y espacios periféricos con los que se amplía la incidencia de la red.

\section{Referencias}

Aguilera, M. (2013). La conciencia red de la multitud conectada: la mente colectiva del 15M. En J. Toret (ed.), Tecnopolítica: la potencia de las multitudes conectadas (pp. 115-132). Barcelona: UOC.

Aragón, P., Congosto, M. L. y Laniado, D. (2013). Evolución del sistema red a través de topología de redes. En J. Toret (ed.), Tecnopolítica: la potencia de las multitudes conectadas (pp. 93-99). UOC.

Baer, H. (2016). Redoing feminism: digital activism, body politics, and neoliberalism. Feminist Media Studies, 16(1), 17-34.

Boix, M. (2015). Desde el ciberfeminismo hacia la tecnopolítica feminista. Revista Pillku, 18, https://pillku.org/article/desde-el-ciberfeminismo-hacia-la-tecnopolitica-fem/ 
Boyd, D., and Crawford, K. (2012). Critical questions for big data: Provocations for a cultural, technological, and scholarly phenomenon. Information, Communication \& Society, 15(5), 662-679.

Fraser, N. (1990). Rethinking the public sphere: A contribution to the critique of actually existing democracy. Social text, 25/26, 56-80.

Granovetter, M. (1983). The strength of weak ties: a network theory revisited. Sociological Theory, 1, 201-233.

Hawthorne, S., and Klein, R. (1999). Cyberfeminism. Melbourne: Spinifex Press.

INEGI. (2017). Encuesta Nacional sobre Disponibilidad y Uso de Tecnologías de Información en los Hogares. Ciudad de México: Instituto Nacional de Estadística, Geografía e Informática.

Inmujeres CDMX. (2016). Ciudad segura y amigable para las mujeres y niñas. Ciudad de México: Instituto de las Mujeres de la Ciudad de México.

Korol, C. (2007). Hacia una pedagogía feminista: géneros y educación popular. Buenos Aires: El Colectivo América Libre.

Latour, B. (2008). Reensamblar lo social: una introducción a la teoría del actor-red. Buenos Aires: Manantial.

Luchadoras. (2018). La violencia en línea contra las mujeres en México. Ciudad de México: Alianza Internet es Nuestra.

Magallanes-Blanco, C., and Rodríguez-Medina, L. (2016). Give me a mobile and I will raise a community. In Communication and Information Technologies Annual: Digital Empowerment: Opportunities and Challenges of Inclusion in Latin America and the Caribbean (pp. 315-343). Emerald Group Publishing Limited.

Melucci, A. (1999). Acción colectiva, vida cotidiana y democracia. México: El Colegio de México.

Negt, O. (2007). L'espace public oppositionnel. Paris: Editions Payot.

Núñez Puente, S. (2011). Activismo y colectivos en red: praxis feminista y violencia de género. Asparkía. Investigación feminista, 22, 85-98.

Plant, S. (1998). Ceros + Unos. Mujeres digitales y la Nueva Tecnocultura. Barcelona: Destino.

Rancière, J. (1996). El desacuerdo: política y filosofía. Buenos Aires: Nueva Visión.

Reverter Bañón, S. (2013). Ciberfeminismo: de virtual a político. Teknokultura. Revista de Cultura Digital y Movimientos Sociales, 10(2), 451-461.

Reverter, S. (2001). Reflexiones en torno al Ciberfeminismo. Asparkia: investigación feminista, 12, 35-51.

Rovira S., G. (2018). El devenir feminista de la acción colectiva: las redes digitales y la política de prefiguración de las multitudes conectadas. Teknokultura. Revista de Cultura Digital y Movimientos Sociales, 15(2), 223-240.

Sierra, F. (2018). Ciberactivismo y movimientos sociales. El espacio público oposicional en la tecnopolítica contemporánea. Revista Latina de Comunicación Social, 73, 980-990.

Sierra, F., and Gravante, T. (eds.) (2017). Networks, movements and technopolitics in Latin America. Critical analysis and current challenges. New York: Palgrave-IAMCR.

Toret, J. (2013). Tecnopolítica: la potencia de las multitudes conectadas. El sistema red 15M, un nuevo paradigma de la política distribuida. Barcelona: UOC.

Trere, E. and Barranquero, A. (2017). Tracing the roots of Technopolitics: towards a north-south dialogue. En F. Sierra and T. Gravante (eds.) Networks, movements and technopolitics in Latin America. Critical analysis and current challenges. New York: Palgrave-IAMCR. 
Wang, T. (2013). Big data needs thick data. Ethnography Matters, 13, http://ethnographymatters. net/blog/2013/05/13/big-data-needs-thick-data/ 